\title{
Laying down a forking path: Incompatibilities between enaction and the free energy principle
}

\author{
Ezequiel A. Di Paolo ${ }^{1,2,3}$, Evan Thompson ${ }^{4}$, Randall D. Beer ${ }^{5}$ \\ ${ }^{1}$ Ikerbasque, Basque Foundation for Science, Bizkaia, Spain \\ ${ }^{2}$ IAS-Research Center for Life, Mind and Society, University of the Basque Country, Donostia, Spain \\ ${ }^{3}$ Centre for Computational Neuroscience and Robotics, University of Sussex, Brighton, UK. \\ ezequiel.dipaolo@ehu.es
}

${ }^{4}$ Department of Philosophy, University of British Columbia, Canada.

evan.thompson@ubc.ca

${ }^{5}$ Cognitive Science Program, Indiana University, Bloomington, USA.

rdbeer@indiana.edu

\section{Abstract}

Several authors have made claims about the compatibility between the Free Energy Principle (FEP) and theories of autopoiesis and enaction. Many see these theories as natural partners or as making similar statements about the nature of biological and cognitive systems. We critically examine these claims and identify a series of misreadings and misinterpretations of key enactive concepts. In particular, we notice a tendency to disregard the operational definition of autopoiesis and the distinction between a system's structure and its organization. Other misreadings concern the conflation of processes of self-distinction in operationally closed systems with Markov blankets. Deeper theoretical tensions underlie some of these misinterpretations. FEP assumes systems that reach a non-equilibrium steady state and are enveloped by a Markov blanket. We argue that these assumptions contradict the historicity of agency and sense-making that is explicit in the enactive approach. Enactive concepts such as adaptivity and agency are defined in terms of the modulation of parameters and constraints of the agent-environment coupling, which entail the possibility of redefinition of variable and parameter sets and of the dynamical laws affecting a system, a situation that escapes the assumptions of FEP. In addition, the enactive perspective foregrounds the enabling and constitutive roles played by the world in sense-making, agency, development, and the path-dependent diversity of human bodies and minds. We argue that this position is also in contradiction with the FEP. Once we move beyond superficial similarities, identify misreadings, and examine the theoretical commitments of the two approaches, we reach the conclusion that the FEP, as it stands formulated today, is profoundly incompatible with the theories of autopoiesis and enaction. 


\section{Introduction}

The Free Energy Principle (FEP; Friston, Kilner, and Harrison 2006; Friston 2012) has spurred a large body of literature in the sciences of mind. A growing subset of this literature concerns a series of claims about the compatibility between FEP and theories in embodied cognitive science, in particular, the enactive approach (e.g., Varela, Thompson and Rosch 1991; Thompson 2007; Di Paolo, Buhrmann and Barandiaran 2017). Several articles (to date over two dozen) have appeared that make some connection between FEP, autopoiesis, and enaction. More can be expected. We are interested in examining these claims. They are varied and presented with justifications that range from pointing out general similarities to attempting to demonstrate deeper, principled connections between the two approaches. These claims have also been interpreted in different ways. Some welcome the prospect of compatibility as positive to both the enactive and FEP positions, leading potentially to a more powerful and unified theory of embodied cognition. They speak of elaborations, interpretations, extensions, syntheses, etc. (e.g., Bruineberg, Kiverstein, and Rietveld 2018). Others think FEP may serve to reconcile enaction with positions enactivists criticize, such as modern versions of representationalism (Clark 2015; Constant, Clark, and Friston 2021; Wiese and Friston 2021). Others adopt a different tone that suggests that FEP has overcome the limitations of the enactive approach, or subsumes or absorbs autopoietic and enactive theories (e.g., Allen and Friston 2018; "FEP subsumes autopoiesis", Korbak 2021, 2747; "FEP provides an implementation of enactivism, and in a sense supersedes or absorbs classical (i.e., autopoietic) formulations," Ramstead et al. 2021, 59). Finally, in contrast to the latter, some propose that enactive ideas can be used to fill in gaps and resolve problems in the FEP framework (Kirchhoff and Froese 2017; van Es and Kirchhoff 2021).

Given this variety, it is difficult to examine this literature as a coherent whole. Moreover, claims continue to change, so that sometimes important details absent in one piece of work are recognized as relevant in another. Nevertheless, we think that there are a few core shared claims that do not seem to change. In particular, there is the claim that the extension of FEP from its original domain of application in neuroscience to living systems in general serves as the basis of a general theory of the relation between biology and cognition, something that resonates with enactive discourse about the continuity between life and mind (Thompson 2007). A more specific common claim concerns the need to attend to the active role of the cognitive agent in engaging the world by following vital norms. This claim underlines the close connection between internal processes, processes in the environment, 
the agent's activity, and the agent's viability. At first sight, enactivists would agree with these claims. But are these parallels merely superficial? The literature we examine here claims that the connection between the two perspectives runs deep. We disagree. We will argue that the apparent compatibilities are based on quick readings, or even misreadings, of enactive ideas and what they entail, while central aspects of autopoiesis and enaction are left unacknowledged.

Clearing up misinterpretations may move the discussion forward, but will not by itself resolve the deeper tensions and incompatibilities between FEP and enaction as theories of cognition. Many commentators focus on what they see as the main source of tension, which has to do with the representational and cognitive-loaded bias that instantiations of FEP and related ideas tend to adopt, particularly in the form of predictive processing models (Hohwy 2013). They argue that FEP admits wider interpretations of terms such as "model" and "inference," and that these interpretations are compatible with enaction's rejection of internalism and computationalism. While these arguments have merit, we think they miss much more basic tensions between the two theories. These tensions have to do with how the enactive approach conceives of agents as precarious, self-constituted entities in ongoing historical development and capable of incorporating different sources of normativity, a world-involving process that is co-defined with their environment across multiple spatiotemporal scales and together with other agents. The enactive view, as we shall explain, is at odds with universalizing the ideas of non-equilibrium steady states and Markov blankets that serve as key assumptions in FEP. Underlying these worries are divergent fundamental conceptions of materiality and temporality. These incompatibilities lead to a series of forking claims about embodied agents in general, claims that take on a sharper contrast in the case of human beings. Nonrepresentational interpretations of FEP do not rejoin these divergences.

Before we proceed, a couple of caveats. We do not intend to critically evaluate FEP altogether (for this, see, e.g., Baltieri et al. 2020; van Es and Myin 2020; Biehl, Pollock and Kanai 2021; Colombo and Wright 2018; Litwin and Miłkowski 2020; Raja et al. 2021; van Es 2020). This would be too large a task for a single paper, and since FEP is an evolving theory, it would be very difficult to offer a fair general critique. We are exclusively concerned with examining claims about the compatibility between FEP and enaction, or claims of useful complementarities between the two approaches and whether enough inter-theoretical common ground exists to support them. Nor do we reject specific applications of FEP ideas or techniques to make sense of empirical data (Walsh et al. 2020). Insofar 
as they offer useful methods for analysis, FEP and predictive processing models can provide helpful tools in specific cases. Our discussion concerns FEP as a universal approach to explaining life and mind. It is at this level that we find significant and hard to reconcile differences with the enactive approach.

\section{An enactive FEP?}

Enactive readings of FEP and claims of compatibility with ideas such as autopoiesis are varied. Some attempt principled unifying arguments (Ramstead et al. 2021), others provide readings of FEP from enactive and ecological perspectives (Bruineberg, Kiverstein and Rietveld 2018), and others simply remark on apparent resonances and similarities (Clark 2015).

Despite this variety, the motivations for these diverse claims seem fairly aligned. We can roughly describe them as a concern to establish embodied-cognition interpretations of FEP that do not necessarily entail a traditionally internalist or representationalist perspective (as predictive processing models tend to do, Hohwy 2013), or that at least recast notions of representation in action-oriented terms (Clark 2015). Some authors are also motivated by the potential extensions of FEP, originally formulated within the confines of neuroscience, to a wider range of biological phenomena-indeed, to the status of a general principle of theoretical biology and psychology. Demonstrating compatibility with the enactive approach, where the continuity between life and mind has been emphasized, may be considered as a step towards this goal.

Because of these aligned motivations, it makes sense to look at this literature as a whole. We group its claims into two broad groups. We are aware that we may miss some nuances, but we do not think these will be central to our purposes.

In particular, we are concerned with the following two groups of ideas: (1) proposals for a link between FEP and the theory of autopoiesis, and (2) discussions of various compatibilities between FEP and enactive ideas.

We present only selected examples in this section and offer a critical analysis in the next two sections. We will not occupy ourselves with other discussions that point to broad similarities between FEP and 
"enactive-like" ideas (e.g., situated embodied activity, action-perception co-dependence) that are shared by various other approaches (e.g., Pezzulo et al. 2015).

It is important to keep in mind that the two groups are obviously related and the division is merely one of convenience. The unspoken assumption at play is that the enactive approach has developed historically from readings and elaborations of the ideas first presented in the classical theory of autopoiesis (Maturana and Varela 1980,1987), and that to this day many central concepts in enaction find their roots in this theory. However-and this may be a confounding factor that we will discuss later-the enactive approach is in many ways a branching development of the theory of autopoiesis and is critical of important aspects of classical autopoietic theory (e.g., Di Paolo 2018). Contemporary defenders of classical autopoietic theory, in turn, have criticized the enactive approach precisely for this reason (e.g., Villalobos and Ward 2015).

Our first group concerns the goal championed by Karl Friston and others of extending the reach of the FEP to biology (and eventually to other domains). This work discusses FEP's relation to the theory of autopoiesis and concerns enactive ideas only indirectly. Nevertheless, its claims form a kind of backbone for work looking at enaction, the reasoning being that enactive theory is an elaboration of the theory of autopoiesis.

Friston (2013) provides an early claim concerning the relation between FEP and autopoiesis (for more up-to-date versions of this claim, see Parr, Da Costa, and Friston 2019; Ramstead et al. 2021; Wiese and Friston 2021). This paper describes the applicability of FEP to biological systems (see also Friston 2012) by explicitly referring to the concept of autopoiesis.

Briefly, autopoiesis is a property of the organization of living systems (Maturana and Varela 1980, 1987). Maturana and Varela (1980) originally postulated it as the defining property, necessary and sufficient for the living organization, but subsequent researchers have argued that autopoiesis is necessary but not sufficient for the living organization (Bourgine and Stewart 2004; Bitbol and Luisi 2005; Di Paolo 2005; see Thompson 2007 for discussion). "Autopoiesis" means that living systems are organized as networks of biochemical processes such that two conditions obtain: (1) (selfproduction) the operation of the processes in the network regenerates the set of relations between processes in the network; and (2) (self-distinction) the network emerges as a distinct topological unity 
in the domain of biochemical interactions. An autopoietic system is therefore materially self-producing and self-distinguishing. Due to the circularity (recursive self-production) to which this idea refers, Maturana and Varela (1980, 1987; Varela 1979) described an autopoietic system as having "organizational closure" or "operational closure." We will come back to these ideas in the next section.

According to Friston (2013), if we assume that a random dynamical system is ergodic, it will tend to evolve towards a regime where the probability density of its states (the chance that a particular state will be visited) does not change over time. If the density of states is unchanging, that is, if the system reaches a non-equilibrium steady state, one can derive a series of relations between internal and external variables. The distinction between these sets of variables is defined by assuming the presence of a Markov blanket that statistically "insulates" one set from directly affecting the other. Instead, effects propagate through action and sensory variables that define the blanket and mediate between internal and external variables. These relations are obtained formally and demonstrate the central claim that "any ergodic random dynamical system that possesses a Markov blanket will appear to actively maintain its structural and dynamical integrity" (Friston 2013, 2, emphasis removed). It is the maintenance of structural and dynamical integrity that Friston sees as the key property of biological systems, from which he conjectures that, to exist, organisms must be ergodic (or at least converge to a non-equilibrium steady state, as the claim has been recently amended, e.g., Parr et al. 2019) and must be in possession of a Markov blanket. ${ }^{1}$

Friston refers to autopoiesis as an attribute of biological self-organization and claims that it can be derived from his analysis. He sees the maintenance of a steady state as a kind of structural integrity;

\footnotetext{
${ }^{1}$ Friston's argument bears a resemblance to an idea presented by W. R. Ashby (1962). "So the answer to the question: How can we generate intelligence synthetically? is as follows. Take a dynamic system whose laws are unchanging and single-valued, and whose size is so large that after it has gone to an equilibrium that involves only a small fraction of its total states, this small fraction is still large enough to allow room for a good deal of change and behavior. Let it go on for a long enough time to get to such an equilibrium. Then examine the equilibrium in detail. You will find that the states or forms now in being are peculiarly able to survive against the changes induced by the laws. Split the equilibrium in two, call one part 'organism' and the other part 'environment': you will find that this 'organism' is peculiarly able to survive against the disturbances from this 'environment." (ibid. 272). This resemblance may explain Friston's insistence on using the language of "appearances" (e.g., internal states appearing to minimize energy, appearing to engage in active inference, and appearing to model the external world, to mention just the instances in the abstract in Friston 2013). Ashby's exercise, like Friston's, is ambiguous between deflating the notion that there is a real distinction between organism and environment, when in fact the boundary is drawn by the observer (or the choice of one of possibly many Markov blankets), and suggesting that to speak of actual distinctions and of their appearances is ultimately the same thing, a view that enactivists tend to reject.
} 
and he sees such structural integrity as a form of homeostasis sustained by the system appearing to act against the dispersion or entropy of its states (Friston 2013, 5). The same argument is repeated in more recent publications (e.g., Parr et al. 2019; Wiese and Friston 2021). The parallel is reaffirmed elsewhere by suggesting that the Markov blanket plays the role of the autopoietic system's boundary-subserving processes of self-distinction. Allen and Friston (2018) further claim that "One can formulate this in another way; the organism's internal states constitute probabilistic beliefs about what actions are the most likely to provide evidence for the organism's existence (survival)" (ibid. 2474), and they state that "This notion is at the heart of autopoietic views of life and mind, insofar as it induces a deeply circular causality between internal and external states, to provide a normative principle by which to understand all action and perception" (ibid. 2474).

The central claim of these arguments is that random dynamical systems with one or several Markov blankets that converge to a non-equilibrium steady-state regime conserve themselves in that regime (or appear to do so depending on the perspective) by minimizing free energy, and that these conditions are equivalent to the condition of a system being autopoietic.

As we discuss later, Friston's (2013) use of the concept of autopoiesis is rather loose. He does not attempt to substantiate his claim by considering the definition of autopoiesis; instead, he bases the claim on a broad analogy between the conservation of systemic properties in the random ergodic systems he proposes and in autopoietic systems. Further elaborations of his argument move into more specific enactive territory and lean more explicitly on ideas such as operational closure, adaptivity, and sense-making (Di Paolo 2005, 2009; Thompson 2007). For instance, Kirchhoff et al. (2018) discuss the significance of the concept of the Markov blanket. After pointing out that metabolic cell production is a process enabled by the existence of cellular boundaries, the authors conclude that "living systems can therefore be construed as a process of boundary conservation, where the boundary of a system is its Markov blanket" (ibid. 6). The dependencies induced by the Markov blanket act as a "kinetic barrier" that keeps the system "far removed from thermodynamical equilibrium." A Markov blanket does not fully isolate the system: "external states may influence internal states even if the former are not constitutive parts of an operationally closed system" (ibid). Ramstead et al. (2021) draw similar analogies between Markov blankets and the boundaries of (unicellular) organisms. More specifically, they claim in agreement with Kirchhoff et al. that "it is fairly straightforward to establish 
that the Markov blanket formalism provides a statistical formulation of operational closure" (Ramstead et al. 2021, 55).

Our second group in the literature we are discussing corresponds to arguments in favour of a broad spectrum compatibility between FEP and enaction, not just the concept of autopoiesis, though in all cases we see the work in the first group referenced uncritically. For instance, Bruineberg, Kiverstein, and Rietveld (2018) accept that FEP is applicable to biological systems, but they reject its association with Helmholtzian approaches, in which perception is mediated by unconscious inferences about the causes of sensation. A typical version of such approaches is the postulation of predictive processing models implemented in the brain. Bruineberg and colleagues argue that FEP is more general and one need not be committed to a Helmholtzian perspective (though even when conceiving of strictly biological integrity in terms of the FEP, Friston talks about active inference or the semblance of it). The authors articulate an ecological-enactive interpretation of FEP, avoiding the Helmholtzian separation between organism and environment, and placing the locus of active inference in the whole situated organism and not its brain. They offer charitable interpretations, such as construing the internal dynamics of active inference as states of action-readiness or expounding Friston's claim that an organism embodies an optimal model of its ecological niche (Friston 2011) in terms of the organism possessing adequate skills that allow it to reach conditions of grip. ${ }^{2}$ These interpretations are indeed more palatable to enactivists and ecological psychologists than the Helmholtzian picture.

Applying a similar ecological-enactive interpretation of FEP to questions concerning the self and the feeling of mineness, Kiverstein (2020) offers a more detailed comparison with enactive technical concepts. Like Ramstead et al. (2021), he describes the concept of autonomy in terms of precarious operational closure and suggests that this concept can be cashed out in FEP terms. According "to the free energy principle, the autonomy of living systems is a consequence of the inferential processes of free energy minimisation" (Kiverstein 2020, 565), and "the free energy principle tells us how living systems might sustain their own operational closure under precarious conditions in their dynamic coupling with the environment" (ibid. 566).

\footnotetext{
${ }^{2}$ Recent interpretations of Friston's claim de-emphasize the idea that the organism implements or is itself a model of its environment, and argue instead that these models correspond to descriptions available to the observer (see, e.g., Baltieri et al. 2020; van Es 2020).
} 
Kiverstein also makes reference to key aspects of the enactive concept of sense-making: "Any system that has autonomy will also qualify as an agent that has its own individual point of view upon the world (Di Paolo et al. 2017). Relative to this point of view the environment has affective significance in terms of how it bears on the organism's self-produced identity. Organisms enact values, purposes and norms which are of their own making in the sense that they originate in processes of selfindividuation (i.e. free energy minimisation) to which the organism owes its existence. ... Perception and action are thus laden with affect" (Kiverstein 2020, 566). Except for the parenthetical reminder that he accepts the equating of self-individuation and free energy minimization, Kiverstein in these passages does not seem to be arguing for a parallel between enaction and FEP as much as for what an enactive interpretation of sense-making could add to FEP.

Kiverstein also suggests that a more embodied and situated understanding of active inference should be in terms of readiness for action, and he uses recent developments in enactive theory to expand on what this idea means. "As the organisation of the autonomous system becomes less bound to its immediate metabolic needs, so the possible meaningful relations the organism can stand in to the environment becomes less tightly bound to the here and now. The organism becomes sensitive to tendencies and trajectories that constitute the dynamical configurations of the organism-environment system, and their consequences for its precarious existence ... The nervous system can then be thought of as generating and sustaining stable and recurrent patterns of sensorimotor engagement with the environment. These patterns of engagement with the environment exhibit just the same properties of operational closure and precariousness as we find in the more basic processes of homeostasis. The argument [here] is that patterns of sensorimotor engagement owe their operational closure to processes of free energy minimisation” (Kiverstein 2020, 566).

In contrast to Kiverstein's attempt at a careful inter-theoretical comparison, other commentators offer their own particular reading of enactive ideas from an FEP standpoint. For example, Clark (2015) defends an embodied and action-oriented construal of predictive processing, which he uses to interpret Varela et al.'s (1991) notion of “enacting a world.” Despite there being several elaborations of this notion in the enactive literature (Thompson 2007; Stewart et al. 2010; Varela 1992, and others), Clark idiosyncratically suggests that one way to interpret phrases like "bringing forth a world" is through processes such as active data sampling used to sustain and update a cognitive agent's predictive models in an action-dependent manner. 
Kirchhoff (2018) follows the thrust of the argument by Friston (2013), adding a few glosses: "FEP adds more to life than AT [autopoietic theory] ... for the FEP, living systems minimize surprise on the basis of embodying a probabilistic model of themselves and their environment. This is a step beyond the appeal to mere AT in explaining the process of self-maintenance” (Kirchhoff 2018, 2526). Following enactive arguments about the insufficiency of bare autopoiesis for sense-making, Kirchhoff states that the FEP "incorporates adaptivity-viz., the future-oriented aspect of cognition-from the very beginning." This point justifies the perspective that, more than just being compatible with autopoiesis, FEP is also compatible with the enactive approach. Adaptivity (Di Paolo 2005) is taken as a complexification of autopoiesis. "This shoehorns nicely with the FEP. Given that the FEP can be shown to apply to systems that are, at least arguably, not cognitive, it follows that mentality arises when organisms minimize free energy to a certain degree-viz., in the context of active inference" (ibid. 2535).

Kirchhoff continues, however, by raising the potential worry that FEP might not fully account for the enactive notion of sense-making and the existential-phenomenological sense of life-mind continuity: "nothing in the FEP is able to account for the constitution of a meaningful perspective" (Kirchhoff 2018, 2535). After describing the idea of sense-making as the most general aspect of mindedness and acknowledging that for enactivists this idea is central to an explanation of life-mind continuity, Kirchhoff asks whether this concept can fully be captured by the minimization of free energy. This is one of the few instances in the literature we are examining where a potential incompatibility (rather than a mere difference) between FEP and enaction is explicitly signaled: "if the FEP gives up on sensemaking, then how can it explain what is central to life and mind?" (2536). Kirchhoff observes that FEP does not negate the idea of sense-making (so the relation between them is not so much compatibility as non-incompatibility), and that this idea has itself been criticized by so-called radical enactivists (Hutto and Myin 2013) for possibly entailing that basic cognition has meaningful content (content subject to correctness conditions), contrary to the nonrepresentationalist aims of the enactive approach (but see Thompson 2018 for a critical response to radical enactivism). In a strange reversal of roles, Kirchoff argues that if there were this entailment, then the concept of sense-making, and hence the enactive approach, would be in tension with the nonrepresentational, leaner version of FEP he defends. Here an enactive explanation of meaning moves from something that FEP as a theory of the mind lacks to something it should not have because it seemingly invites representations back. 
Elsewhere we see similar worries about FEP's potential lack of cognitive specificity, as in Kirchhoff and Froese (2017), who suggest that enactive theory can constrain overly broad interpretations of FEP. As we mentioned, Friston's (2013) argument applies to any random ergodic dynamical system with a Markov blanket, and so the conclusions should be valid for a wide range of systems, including systems traditionally considered non-living or non-cognitive. Kirchhoff and Froese argue that Friston should therefore be committed to a view that sees mind everywhere, as part of any system that minimizes free-energy, including non-biological ones (or, given some kind of life-mind continuity, to a view that finds both life and mind nearly everywhere). Given the existence of systems, such as a candle flame, or in Bruineberg et al.'s (2018) example, two synchronized pendulum clocks, whose stable features are more parsimoniously explained through dissipative self-organization or dynamical couplings than by supposing that internal variables are performing active inferences, Kirchhoff and Froese are right to worry about a thinning out of the explanatory power of the FEP. They suggest that life and mind should be seen as strongly continuous, and that this continuity places cognitive specificity demands that FEP does not meet. To remedy this problem, they suggest that enactive versions of life-mind continuity, defined in terms of autonomy, adaptivity, and sense-making, should be used to constrain an otherwise too liberal FEP.

In this quick exposition we have seen a sample of how FEP, autopoiesis, and enaction have been discussed almost exclusively in terms of important compatibilities. These compatibilities are of different kinds, ranging from broad analogies between free energy minimization, Markov blankets, and aspects of autopoiesis to more elaborate readings using enactive concepts such as autonomy, adaptivity, and sense-making. These compatibilities serve to indicate the possibility of a more detailed inter-theoretical relation or to supplement what some authors see as problematic aspects of FEP as a candidate universal approach to life and mind. We find very few instances where potentially unresolvable incompatibilities are mentioned, making things look as if, except for some details and clarifications, there is a broad consensus about the match between the approaches.

Before we discuss why we strongly disagree with this picture and we examine deeper problems that have not been raised by any of these authors, we will mention a few misreadings that we consider important to clarify for this and future discussions. 


\section{Misreadings}

The enactive approach owes much to the classical theory of autopoiesis but it has never been identical with it. Enaction inherits several concepts from this theory, and more importantly, a sensitivity to the need to provide operational definitions of its key ideas. As well as serving as a constraint on theory development, such definitions should also facilitate inter-theoretical debates. Concepts such as autopoiesis, autonomy, operational closure, sense-making, and agency are defined in operational terms in the enactive literature to avoid vague interpretations. It is surprising that very few attempts to link FEP, autopoiesis, and enaction have taken advantage of these operational definitions and that technical terminology is sometimes used loosely or interpreted narrowly. This sloppiness results in a series of misreadings that, as we argue later, signal deeper incompatibilities between the approaches. Here we indicate some of these misreadings.

\subsection{Autopoiesis}

Let us start by re-stating the classical definition of autopoiesis (after Maturana and Varela 1980, 7879):

An autopoietic system is organized (defined as a unity) as a network of processes of production (transformation and destruction) of components which:

(i) through their interactions and transformations continuously regenerate and realize the network of processes and relations that produces them; and (ii) constitute the system as a concrete unity in the space in which the processes exist by specifying the topological domain of its realization as a network.

We call the first and second requirements "self-production" and "self-distinction," respectively. It is possible to show that both requirements are dialectically related to each other by using the concept of adaptivity (Di Paolo 2005, 2018), which is central to recent enactive literature (Thompson 2007; Di Paolo and Thompson 2014). We return to this concept below.

A few things should be immediately apparent from this classical definition. Autopoiesis is not defined as self-organization or persistence over time (Friston 2013). The autopoiesis definition describes the 
organization of a system that jointly fulfils the requirements of self-production and self-distinction. A system may self-organize and not be autopoietic. It may persist over time and not be autopoietic. And it may individuate spontaneously and still not be autopoietic. The dynamic processes and conditions that form a crystal in a supersaturated liquid solution result in individuation, self-organization, and persistence over time but they do not constitute an autopoietic system. Does autopoiesis entail individuation, self-organization, and persistence over time? The answer will be a qualified yes for most concrete cases. But the presence of these properties does not, in any combination, entail autopoiesis. When present, these properties are realized in specific ways in autopoietic systems, i.e., by fulfilling the requirements of self-production and self-distinction.

To clarify these relations, classical autopoietic theory introduces a distinction between the structure and the organization of a system (Maturana and Varela, 1980). The structure is the system's actual realization, the concrete components that constitute a system and the actual and concrete relations between them. The system's organization is the abstract set of relations that define the system as belonging to a class. Autopoiesis is the description of a class of systems, i.e., a description of the organization that defines this class. Concrete autopoietic systems may be instantiated in a wide variety of structures, and a given structure may belong to more than one class of organization (Fido is a dog, a mammal, a living organism). Structures may also change over time, even if the organization is invariant (Fido was a puppy, is an adult hound, will be a lazy senior). The distinction between structure and organization, as well as other technical concepts, such as structural coupling and operational closure, are further clarified by Beer $(2015,2020)$ using a toy model that reveals other subtleties about these ideas, such as relevance of their time-extendedness.

To come back to our question, does autopoiesis entail individuation, self-organization, and persistence over time?, it should now be clear that the answer depends on whether we are talking about structure or organization. For instance, the autopoietic organization of a living organism persists over time as long as it lives, but its structure may not. Again, statements about the structure of a system do not obviously translate into statements about its organization, and vice versa.

If it is to be more than a loose analogy, Friston's (2013) proposal that random dynamical systems with a Markov blanket in a non-equilibrium steady regime are autopoietic needs operational clarification. 
Are the conditions met by these systems both necessary and sufficient for autopoiesis, especifically for the organizational requirements of self-production and self-distinction?

Autopoiesis entails the conservation of biological organization and an adaptive relation to the environment, a relation that allows the autopoietic system to remain viable. Conservation of organization has sometimes been illustrated in the classical autopoietic literature as a special form of homeostasis, lending some credence to Friston's interpretation. For instance, “an autopoietic machine is an homeostatic (or rather a relations-static) system which has its own organization (defining network of relations) as the fundamental variable which it maintains constant" (Maturana and Varela, 1980, 79). The qualification ("relation-static") should be read as a warning that we are not talking here of homeostasis in the traditional sense. Indeed, the idea of an autopoietic organization as a regulated variable is problematic and not generally accepted. At the organizational-as opposed to the structural-level there is no gradient to the condition of being or not being autopoietic that could be thus regulated (Di Paolo 2005).

We find in Friston's and later arguments a collapse of the distinction between structure (to which his sense of homeostasis refers) and organization (to which the autopoietic sense of homeostasis refers). The structural integrity of systems with a Markov blanket as they reach a non-equilibrium steady regime bears some resemblance to the idea of a system that actively conserves its organization. But it is not the same idea. To establish this point, the question we need to answer is whether either idea entails the other. As in the case of crystal formation, the random dynamical systems postulated by Friston show properties that may be shared by autopoietic systems but are not the properties that define them as autopoietic, i.e., they are also properties shared by non-autopoietic systems. They also show properties that not all autopoietic systems necessarily possess.

A simple contrast with the definition of autopoiesis can verify these claims. Several discrepancies become apparent. There is no obvious equivalent in Friston's systems to the network of processes that through transformations regenerate the conditions of its own production. In fact, the assumptions (of an invariant density of states and the presence of a Markov blanket) are such that no regeneration is needed after the initial event, the organization of the system simply endures. It is also unclear in what sense the components of the systems, e.g., its Markov blanket, are produced by other processes in the system, instead of just being there. Furthermore, from the definition of autopoiesis, we can see 
that structural integrity and even structural homeostasis are insufficient for autopoietic organization. Friston himself may be thinking of this problem when he recognizes that more than entropy minimization is needed to distinguish a biological system from a petrified stone (Friston 2013, 11). In addition, much of the subsequent literature complements Friston's formal argument with intuitive properties that living systems possess (such as being far from equilibrium and actively engaging the environment). But these properties simply raise new questions, such as to what extent they are entailed by free energy minimization and to what extent they entail autopoiesis.

Another discrepancy that emerges from not paying sufficient attention to the structure/organization distinction is that there is no principled reason why it is not possible for a system to be autopoietic (have an autopoietic organization) without having the structural properties postulated by Friston (invariant density of states and Markov blankets). As we discuss in Section 4, there is ample evidence that living systems undergo processes of transformation, not only during development, but also at behavioral and even faster timescales, and that in general they do not find themselves permanently in a steady state, dynamically or materially. Friston and other colleagues qualify the steady-state requirement by talking about local or near ergodicity, but such a move raises more questions than it answers, because to qualify as any sort of "principle" one would have to postulate that such conditions almost always obtain (but do they?), and that when they do not obtain, these situations are not of particular biological or cognitive interest (but are they?). These moves do not change the fact that there is no principled reason why the absence of the structural properties postulated by Friston and colleagues would prevent the system from fulfilling the organizational requirements of self-production or self-distinction.

In summary, the concept of autopoiesis has been loosely interpreted and indeed often misread to different degrees by Friston and colleagues. Attending to the operational definition of autopoiesis and the distinction between structure and organization, it is possible to show (1) that Friston's systems do not entail autopoietic organization; and (2) that autopoiesis may be realized by structures that do not have the properties assumed by Friston (as we will discuss further in Section 4). In other words, FEP systems and autopoiesis do not entail each other. At most, in some instances there may be a contingent and temporary overlap between the two classes of systems. 


\subsection{Boundaries}

The concept of operational closure under precarious conditions (explicated in Di Paolo and Thompson 2014) underpins the enactive idea of autonomy (derived from Varela 1979), another technical term that extends the notion of autopoiesis to more general domains:

[An] autonomous system is defined as a system composed of several processes that actively generate and sustain an identity under precarious circumstances. In this context, to generate an identity is to possess the property of operational closure. This is the property that among the conditions affecting the operation of any constituent process in the system there will always be one or more processes that also belong to the system. And, in addition, every process in the system is a condition for at least one other constituent process, thus forming a network (Di Paolo 2009, 15).

In other words, the processes that constitute an operationally closed network relate to each other such that they form a mutually enabling set of relations. They do so under precarious conditions, meaning that in the absence of these mutually enabling relations, the same processes would tend to run down. No component is, in other words, strictly self-standing in the absence of the whole network.

Operational closure does not mean that processes external to the network cannot influence those that belong to it, or that processes in the network cannot influence processes external to it. Nor does it mean that these influences cannot have an enabling character, i.e., by being causally necessary for internal processes to continue to exist. In other words, an operationally closed system is open not only to informational external influences but also to all kinds of material and formative relations, including, in the case of living systems, the transport of heat, mass, biomatter, genetic material, microorganisms, and so on. Internal processes can literally depend on such enabling exchanges to continue to operate.

Ramstead et al. (2021) and Kirchhoff (2018) make reference to operational closure. These authors see in this concept an enactive analogue of a Markov blanket. For Ramstead et al. (2021, 555) "it is fairly straightforward to establish that the Markov blanket formalism provides a statistical formulation of operational closure.” For Kirchhoff (2018), FEP accommodates, through the idea of Markov blankets, the requirement of operational closure and goes beyond it. Confusingly, Kirchhoff, following Friston 
(2013), also states that it "can be shown that Markov blankets operate in much the same way as a cell boundary" (Kirchhoff 2018, 2527; see also Kirchhof et al. 2018, 6; Allen and Friston 2018, 2473). We also read that a "cell therefore has a Markov blanket —its plasmalemma [cell membrane]" (Ramstead et al. 2021, 551). More complex organisms are described as bounded by an ensemble of nested Markov blankets (Kirchhoff et al. 2018). In addition, Markov blankets are said to act as the epistemological and ontological boundaries of cognitive, not just biological, systems (Ramstead et al. 2021, 551). Similarly, Kirchhoff and Kiverstein (2019) propose using the formalism of Markov blankets to delineate flexible boundaries for the mind.

Such statements are speculative and perplexing. They again conflate very different ideas by confusing the organization and the structure of living and cognitive systems. We see no obvious relation between operational closure (an organizational statement about how a network of processes actively produces and distinguishes itself) and Markov blankets (a statement about statistical conditional independence between sets of variables). Very few commentators seem to have remarked on this discrepancy (e.g., Bruineberg and Hesp 2018, 38). As we have said, an operationally closed system is open to all kinds of interactions and exchanges with the external environment, as long as its organization is not destroyed. These exchanges are typically regulated by the system, but not always (think of exposure to ionizing radiation or the effects of gravity), and not always successfully (think of a viral infection). Such breakdowns are not necessarily fatal, meaning the condition of closure can still be sustained. In such cases, and even when exchanges are successfully regulated, it seems unreasonable to propose that internal variables remain statistically independent of external states. Such independence is by definition impossible if the exchanges are enabling, i.e.. depended upon, as in the case of horizontal gene transfer through which bacteria spread antibiotic resistance, or simply in cases of photosynthesis, osmosis, respiration, and so forth.

Moreover, there is no obvious or principled relation between operational closure and biological or cognitive structural "boundaries" (nor are there obvious relations between the boundaries of biological and cognitive systems, as discussed in Di Paolo 2009). If we know how the organization of an operationally closed system is instantiated, we could in principle point to processes that belong to it and processes that do not. But this epistemic operation is not replaceable by the act of pointing to a physical structure, such as a cell membrane. Maintaining the condition of self-distinction can lead to processes that regulate complex, structural and spatial boundaries, as in the case of cell membranes, 
but also to other processes, such as the prokaryotic CRISPR-Cas immune system that defends unicellular organisms from viruses (Rath et al. 2015). Nevertheless, importantly, these processes, including membranes, are not in any organizational sense at the boundary of the operationally closed system; rather, they are part of it, they are in it.

In sum, the status of the relations between very different concepts, such as the systemic distinction enabled by operational closure, the structural processes that subserve self-distinction, the presence of actual spatial boundaries, and the conditional statistical independence between internal and external variables (Markov blankets) is anything but "fairly straightforward." These are very different ideas, and examples can be found that instantiate one and not the other. If there are any principled expected entailments between these processes and ideas, it is a matter for a much more careful investigation.

\section{Points of tension between the two approaches}

As we have said, articles attempting to establish some kind of connection between FEP and enaction continue to appear as we write these lines. Some claims seem to change in tone and detail, but so far we have not seen many cases in which the misreadings we have indicated are not present in some form or other. In this section, we raise points of deeper tension between the two perspectives. This task is not helped by the ongoing revisions and refinements to the claims that FEP and the enactive approach are compatible, or have sufficient common ground to be fruitfully complementary. Nevertheless, we will attempt to describe what we see as incompatible assumptions between the two approaches and incompatible directions in their respective research programmes. To be as fair as possible, we will do this by attending to the more recent published versions and variants of these claims.

\subsection{Non-equilibrium steady states versus history}

The work presented in Friston (2013) is central to practically all the proposals comparing FEP and enaction. It continues to be cited approvingly to this day. Although it has been argued that the formal argument presented in this work has technical problems (e.g., Biehl et al. 2021, see also Friston, Da Costa and Parr 2020), we will not focus on these problems. Instead, we question whether the 
assumption of non-equilibrium steady states, which is central to this argument, is widely applicable to biological and cognitive systems, or whether, on the contrary, it makes sense only under restricted conditions. In addition, we will ask whether the enactive approach operates under similar restrictions.

Originally, the assumption we target was presented as the requirement that the random dynamical systems in question must be ergodic, or nearly ergodic (meaning: sufficiently ergodic during a period and scale of observation). In more recent work, the condition of ergodicity has been replaced by the weaker assumption that the systems in question must reach a non-equilibrium steady state (Parr et al. 2019; Friston 2019). Both assumptions, the stronger and weaker versions, enable the same crucial step in the formal derivation of FEP mathematical relations, namely, the vanishing of the rate of change of density of states in the system. This results in a steady distribution in the probability of visiting particular states even if the variables continue to change over time. Proponents of FEP interpret this step as "formalizing the notion that a system maintains its form over time" (Parr et al. 2019, 6), where the concept of form has been reduced to an invariant statistical property of the distribution of states. This reduction raises the question of how changes in form can be accounted for within this formalization.

Requiring the system to be in a non-equilibrium steady state in order for the formalism to work is a fundamental point of contrast between FEP and enactive theory, one which few researchers have remarked upon. Like organizational approaches in biology (e.g., Longo and Montévil 2014), enaction emphasizes the historical nature of life and mind (even more than the classical theory of autopoiesis does; see Thompson 2007; Di Paolo 2018; and Varela 2011/1994³). Fundamental enactive ideas, such

${ }^{3}$ In 1994, Maturana and Varela wrote individual prefaces to a new edition of De Máquinasy Seres Vivos, the first book on their joint work on autopoiesis. In his preface, Varela $(2011 / 1994)$ acknowledges that an important criticism of this early work was that autopoiesis seemed to imply a form of solipsism. Maturana and Varela had replaced the idea of mental representations with the idea that the environment triggers perturbations to the ongoing and operationally closed processes of the living system. This proposal included the notion of structural coupling, the idea that the living system and environment act as mutual sources of perturbation, triggering changes of state in each other. Varela notes that this notion seemed to many researchers to be a weaker alternative to the input-output approach of the information-processing metaphor, "because it seems to leave the phenomenon of interaction in a grey area of being a 'mere' perturbation" (ibid. 614). Structural coupling "does not properly take the account of the emerging regularities in the course of a history of interactions ... Over these years I have developed an explicit alternative ... turning the historical reciprocity into the clue of a co-definition between an autonomous system and its environment. I propose to call this point of view in both biology and cognitive science, enaction" (ibid.). History and organism-environment co-definition have been explicit concerns of the enactive approach since its beginnings, and they constitute a change in emphasis and orientation with respect to classical autopoietic theory. 
as the concept of sense-making, operate through mutually defined transformations of agent and environment that can only occasionally be deemed as taking place in a non-equilibrium steady state regime. Rather, these ideas attempt to theorize precisely what happens in transitions between relatively metastable conditions. Varela uses the word "enaction" to refer precisely to such processes that happen "between one behavioral moment and the next" (Varela 1992, 106); and Di Paolo et al. (2017) describe such concrete acts of sense-making as the open transition between microworlds of activity. These key enactive ideas are attempts to describe transitions between different dynamical regimes, and for this reason they are fundamentally incompatible with steady-state assumptions. Linking the idea of form to a statistical (and hence structural) invariant makes explaining such transitions impossible.

This theoretical difference is accompanied by a significant empirical load. The assumption of a nonequilibrium steady regime breaks down in many important cases that are central areas not only of enactive research but also of biology and psychology in general, such as the formation of sensorimotor habits (Di Paolo et al. 2017) and the formation of shared repertoires for coordinated action (Di Paolo et al. 2018). The assumption also breaks down at shorter, behavioral and neural timescales, as in cases of soft assembly and critical agent-environment integration (e.g., Anderson, Richardson and Chemero 2012), or the long-range coordination of neural populations at the moment of perceptual awareness (Varela et al. 2001). At multiple scales, living agents are constantly undergoing regulation in relation to the set of constraints that redefine their structural dynamics (phase spaces) moment to moment. By contrast, systems that maintain non-equilibrium steady-state distributions must eventually forget their history. ${ }^{4}$

Although it may be the case that random dynamical systems reaching a non-equilibrium steady regime tend to maintain their structural integrity by minimizing the tendency to disperse, it does not follow that systems whose organization tends to be maintained must perforce be in such a dynamical regime, a point we have already raised in the previous section. In fact, evidence indicates that in general they are not. Organizations can endure even if structures change. Without attempting to review the many

\footnotetext{
${ }^{4}$ In this context, the relevant difference between an ergodic system (the original FEP formulation) and a system in a non-equilibrium steady regime (the current formulation) is that whereas the former cannot retain in its current state any traces of historical change (the long-term average of states must be the same as averaging the states in an ensemble of systems), systems in steady regimes can retain a historical record of changes undergone before the system reached the steady state (for this reason, the long-term average of such states can be different from the ensemble average). Once it has reached the steady regime, however, the possibility of further retention of historical changes is gone.
} 
cases in biological, neural, and cognitive systems where processes of historical transformation are at play, we can mention some examples. Examples at the biological level are embryogenesis, life-cycle patterns, epigenetic variability, metamorphosis, and symbiosis. ${ }^{5}$ At behavioral levels we observe fluid, critical agent-environment integration across scales in the development of perceptual learning, skill acquisition, expert tool use, and habit formation (Anderson et al. 2012). Finally, at the neurocognitive level, we see developmental plasticity, possibly many-many mappings between neural networks and cognitive functions (Pessoa 2014), and more generally what Anderson (2014) calls "neural reuse," the continued acquisition of new uses for neural circuits in evolution and development without those circuits losing their original uses.

Unlike FEP, the possibility of cumulative historical change is introduced unequivocally in enactive theory through the concept of adaptivity (Di Paolo 2005, 444). Adaptivity is the system's capacity to regulate its states and relation to the environment in ways that result in the avoidance of trajectories that move towards loss of viability. This definition of adaptivity entails that adaptive interventions necessarily modulate the dynamical landscape, for instance, by changing relations to the environment. By definition, this is the only way in which it is possible to alter the tendencies of dynamical trajectories bound to break through the viability boundary in a state-determined system. Adaptivity, as defined and used in the enactive approach, is therefore fundamentally incompatible with the assumption of non-equilibrium steady states, which cannot be obtained if parameters, constraints, and even the system's variable sets are modified in the course of an adaptive event. Thus, two ideas that are key for each approach (adaptivity and non-equilibrium steady states, respectively) are at odds with each other.

Adaptivity makes explicit the aspects of time direction and time granularity of agency (Di Paolo 2005). An adaptive act is in itself not a conservation, but a modulatory deviation from an existing tendency that would lead to eventual loss of viability if left unchecked. An adaptive act takes time, has a particular time-course with different phases, and must occur within appropriate time constraints (given by requirements of speed, deadlines, by the relevant embodied and social norms, and so on). History is made possible by these properties, especially as organisms become more complex, and adaptive acts

\footnotetext{
${ }^{5}$ It is odd to find very few references in the FEP literature to these cases and the potential challenges they offer to the premises of the approach. One exception is Clark (2017), who realizes that cases such as metamorphic insects can be puzzling from a FEP perspective. The solution, he suggests, is to look at whole life cycles as the free energy minimizing strategy of complex organisms. This idea, however, challenges the assumption of nonequilibrium steady states, as Clark himself notes in a footnote (16).
} 
relate to one another in networks of mutual influence and triggering, affecting, moment to moment, the relational constraints that shape the dynamics of the agent. The "historicity" of biological systems (Longo and Montévil 2013) is precisely the cumulative change of dynamical constraints, parameters, and even variable sets throughout a lifetime of changing mutual dependencies between organism and environment (including other organisms).

The FEP's ruling out of historicity (as a consequence of its key assumptions) is thus a central discrepancy between it and the enactive approach. The discrepancy is not minor. The possibility of history is not just implied by enactive concepts but also elaborated in enactive research, particularly to theorize concepts of minimal agency, mastery of sensorimotor contingencies in action and perception, and the (historically and culturally contingent) development of sensorimotor repertoires as the theoretical basis for the concept of sensorimotor agency and linguistic bodies (Di Paolo et al. 2017, 2018). One of the definitional requirements for agency proposed by enaction is that of interactional asymmetry. In dynamical systems terms, this requirement is meant to capture that not only are agent and environment coupled systems, but also that processes in the agent can trigger modulations (changes in parameters and constraints) of this coupling. Again, such modulations of parameters and constraints cannot guarantee the conservation of a non-equilibrium steady state regime. They typically will not, and these changes are what allow the system to avert the potential loss of viability were it to remain in such a regime. As in the case of widespread extended critical transitions in biological systems (e.g., Longo and Montévil 2013), the regulation performed in the acts of an agent can give rise to pathdependent changes on the constraints affecting the dynamical flows of the agent as a whole, essentially altering the configuration of the phase space of organism and environment, and rendering notions such as attractive sets and non-equilibrium steady states inapplicable, except under particular conditions. The enactive concept of agency reaffirms for cognitive systems what Longo and Montévil predicate about biological systems: "Biological processes are more 'history based' than physical processes. Usual physical processes preserve invariants, whereas extended critical transitions [characteristic of biology] are a permanent reconstruction of organization and symmetries, i.e., of invariants" (ibid, 175).

As far as human beings are concerned, evidence strongly suggests that processes of historical change cannot be disregarded. Ergodicity and steady-state regimes in psychology and neuroscience may sometimes make methodological sense in laboratory conditions. But these conditions are not always 
made explicit and these assumptions can lead to systematic problems in interpreting the generality of empirical results. Indeed, several researchers interpret the current crisis of replication in psychology precisely as a crisis provoked by assuming ergodicity by default, a practice that belies the lack of an underlying theory that would grant validity to the assumption for each given case (Molenaar 2004; Molenaar and Molenaar and Nesselroade 2014; Speelman and McGann 2016, Rose et al. 2013).

For instance, there is no forgetting of history in habit formation but rather an actual embodiment of history. We could say that there can be no habit without history, or that history is constitutive of the concept of habit. Enactive literature is very clear about this point: "To say that the habitual body acts as guarantee for the body at this moment is to say that one's lived body is a developmental being thick with its own history and sedimented ways of feeling, perceiving, acting, and imagining. These sedimented patterns are not limited to the space enclosed by the body's membrane; they span and interweave the lived body and its environment, thereby forming a unitary circuit of lived-bodyenvironment" (Thompson 2007, 33).

In addition, history and contingency lead to diversity, which a theory of human bodies and minds cannot afford to leave without a proper theoretical grounding. The beginnings of such a theory is offered in (Di Paolo et al. 2018). Cultural and interpersonal variability in human beings are not statistical noise, but rather necessary consequences of what it means to undergo human becoming. Some events may enter the developmental, cognitive, and emotional history of a person as accidents, but if their effects endure or get amplified, this occurs by triggering a swerve in dynamical paths and reshaping dynamical landscapes. Fluctuations can become locked-in and change the course of subsequent history. None of this entails a loss of the system's integration, but it may entail transformations that redefine skills, sensitivities, meanings, and norms. Widespread biological and psychological phenomena (of the kind FEP proponents intend to cover with their theory, e.g., Veissière et al. 2020) are negated, minimized, or rendered anomalous if the assumption of nonequilibrium steady states is universalized. The idea of a second nature becomes inconceivable.

In its concern with historical change, the enactive approach has provided accounts of perception and perceptual learning in terms of mastery of sensorimotor contingencies linking multiple scales from neural to developmental processes (Di Paolo et al. 2017). The idea of mastery is operationalized using a dynamical theory of equilibration that does not require systems to be in non-equilibrium steady 
regimes to work (Di Paolo et al. 2017). Indeed, some phenomena such as developmental spurts in skill level (Fischer and Bidell 2006) signal changes in dynamical configurations (novel constraints, emergent parameters, changing variable sets). The variability entailed in changing dynamical configurations has been postulated as the origin of motor creativity (Orth et al. 2017), the very idea of which is rendered problematic on the assumption of non-equilibrium steady states. In contrast, all of these processes can be accommodated by the enactive theory of sensorimotor learning, which also accounts for the existence of multiple developmental pathways, path-dependence, and the intrinsic variability found in all kinds of human skills (Kostrubiec et al. 2012; Thelen et al. 1996; Adolphs and Hoch 2019).

A central claim of the enactive approach is that sense-making is an autonomous activity that contrasts with heteronomous information processing. To illustrate the sort of neural processes that underpin sense-making, Varela (1992) and Thompson (2007) make repeated references to the work of Walter Freeman, who explicitly acknowledges history-dependence in the most basic neuroscientific scenario of stimulus processing: "The emerging [neural] pattern ... is a state transition that is induced by a stimulus, followed by a construction of a pattern that is shaped by the synaptic modification among cortical neurons from prior learning. ... Owing to dependence on history, the patterns created in each cortex are unique to each subject" (Freeman 1999, 149-150).

In discussing the fine temporal structure of cognitive action, Varela proposes that coherent patterns of fast neural oscillations emerge at moments of concretion (significance) in action and perception (Varela 1992). This idea has been supported empirically in studies of long-range neural (de)synchronization at moments of perceptual awareness (recognition) and action initiation, evidencing emergent processes that rapidly constrain and free dynamical flows contingently on the actions of the perceiver and her situation (Varela et al. 2001). This perspective on brain function puts the emphasis on neural processes radically altering their dynamics in fluid and adaptive ways so as to meet the demands of a concrete sociomaterial situation (e.g., Anderson 2014; Pessoa 2014; Fuchs 2017). Such rapid moments of high dimensional dynamic expansion followed by lower dimensional coordination is one of the meanings given to the term enaction itself. Accordingly, "the hinge that articulates enaction consists of fast non-cognitive dynamics wherein a number of alternative microworlds are activated. These hinges are the source of both common sense and creativity in cognition" (Varela 1992, 109). The latter remark indicates that these fast neural processes are not just history-dependent (“common sense”) but also history-making (“creativity"). 
The idea of inhabiting and transitioning between microworlds has been further developed by Di Paolo et al. (2017), where the authors also explore the possibility of extended periods of high-dimensional exploration of sensorimotor possibilities when the transition between activities does not occur or for whatever reason is arrested. They call these moments of uncommitted dynamical engagement the zero-mode of activity. Using a network metaphor to describe the functional and structural relations found within a repertoire of sensorimotor schemes, the authors remark "through regional developmental differentiation and integration of schemes, integrated subnetworks can be formed that correspond well to notions such as activities, microworlds, and sensorimotor genres. The structure of the sensorimotor network reflects the history of the agent. In humans the possibilities are open-ended and path-dependent, as we would expect, leading to a way of characterizing otherwise vague concepts such as sensorimotor styles" (Di Paolo et al. 2017, 177-78).

In sum, whether at neural, behavioral, or developmental scales, these enactive ideas all share in common not only the possibility but also the expectation that biological and cognitive systems change in path-dependent ways, not only at some points in time, but also regularly, at different scales, and throughout their lifetime.

The point here is not to defend the merit of these enactive ideas, but rather to highlight the extent to which they substantially differ from FEP. History is central for the enactive approach. History is not just a possibility admitted in theory, but also a thread that has driven much of the theoretical development and current directions of the enactive approach (e.g., see recent articulations of the notion of open-ended human becoming in Di Paolo 2020 and De Jaegher 2019). Again, this marks a fundamental theoretical incompatibility between the enactive approach and FEP at the level of their basic assumptions, as well as a point of divergence between them in their overall research directions.

A possible attempt at making both approaches compatible on the issue of historicity might take the form of an argument that renders historical changes as only apparent and explains them as a consequence of the transitions between existing and pre-established metastable states within what is otherwise a system in a non-equilibrium regime (when seen at appropriately long scales). This is similar to Clark's (2017) idea to regard whole life-cycles as a free energy minimizing strategy. Thus a transition between one microworld and another one, as described in enactive terms and as it occurs in daily life, 
could be described as a switch between metastable "micro-regimes" within the non-equilibrium steady state. This explanation might accommodate some cases of routine activities, but if it were generally the case, it would imply that all of our activities are routine, at least statistically. The steady distribution of states must translate into a steady distribution of transits between metastable micro-regimes. Engaging in novel activities (e.g., learning to drive) or abandoning old ones (because of loss of interest or other factors) cannot be explained in this way.

A more general point should also be mentioned. If the status of FEP as a principle of wide applicability across many scales of biological, cognitive, and cultural phenomena (e.g., Veissière et al. 2020) rests on the mathematical models used to describe it, and they in turn rest fundamentally and (for the moment) unavoidably on the assumption of non-equilibrium steady states, then the FEP cannot really be universal, as is often suggested. It also cannot be sufficiently specific to distinguish life from nonlife (which is a different but related problem that motivates some researchers to seek for links to enaction, e.g., Kirchhoff and Froese 2017; van Es and Kirchhoff 2021).

This problem cannot be solved by admitting that the central assumption of non-equilibrium steady states can sometimes be relaxed or is relative to a timeframe of relevant observation. To admit this is, again, the same as conceding that the FEP does not really have the claimed status of a universal principle. The move begs the question of what happens to biological and cognitive systems in the moments the steady-state assumption does not hold (moments that the FEP is self-restricted from saying anything about). The question that a theoretical biology and a theoretical psychology should be able to answer is when this assumption, and hence the FEP, is sufficiently valid for its application to be useful. If, as seems to be the case, important phenomena (e.g., ontogeny, acquired immunity, the time course of illness or injury and recovery/compensation, skill acquisition, habits, development towards emotional maturity, trauma, personality, language, abstract thought, social interactions, expert use of tools, cultural history, evolution of technology, changes in modes of production, and innumerably other path-dependent processes) fundamentally break this assumption, it does not follow that we should discard FEP ideas as useful research tools, provided we can specify the conditions under which their use makes sense. But FEP cannot answer this question by itself. The specification must come from a theoretically-loaded account that tells us when to expect the non-equilibrium steady-state assumption to hold as a good approximation, and when not to expect this. It goes without 
saying that the same theory cannot adopt this assumption unquestioningly and also tell us as a corollary when it does not hold. So we must look outside the FEP for an answer.

\subsection{Mutual enablement and co-constitution of internal and external domains}

In Section 3, we noted the recurrent and puzzling confusion between Markov blankets and what autopoietic and enactive theories specify as processes of organizational self-distinction. These

processes of self-distinction do occasionally-but not exclusively-take the form of processes of structural boundary formation, regulation, and maintenance. They can also take other forms (e.g, immune responses against invading pathogens, temperature regulation in warm-blooded animals, musculoskeletal systems of tension maintaining bodily shape). In all instances they involve adaptive regulations of material exchanges between organisms and the environment. We can ask whether the confusion between such processes and Markov blankets is symptomatic of another deep tension between the two approaches, a tension in how they conceive of relations between organism and environment and relations between different organisms; whether these relations are merely informational (i.e., contextual), or can also be transactional (e.g., mutually enabling) or even constitutive of biological and cognitive/affective processes. Enactivists assert a strong notion of world-involvement, i.e., processes in the environment play more than informational roles in the constitution and actualization of life and mind (Di Paolo et al. 2017; Thompson and Stapleton 2009). To enact a world of significance entails to engage in actual acts, which are material events with spreading consequences that are both world-changing and agent-changing. Environmental and biological/cognitive processes are mutually enabled and mutually constituted. They interpenetrate at all scales and they coordinate across scales. Can these claims be accommodated by the idea of Markov blankets?

Here we encounter another source of tension between enaction and the FEP, though one not entirely unrelated to the divergences we have just discussed concerning historical processes. Indeed, the issues about historicity and co-constitution of organism and environment are internally related in the enactive approach, whereas they are only contingently related in FEP, and this difference is itself important. Concerns about the conservation of organization are mostly linked to the self-production requirement 
of autopoiesis (the regeneration of the conditions that continuously give rise to the operationally closed network of processes making up the organism). Concerns about barriers, boundaries, and in general about an organism's relation to its environment are mostly linked to the condition of selfdistinction in the definition of autopoiesis. From an enactive perspective, self-distinction and selfproduction are dialectically (internally) related (Di Paolo 2018), that is, they are mutually dependent, though distinct, moments of self-individuation (Varela 1991; Thompson 2007). You cannot have one process and not the other as long as the organism lives, yet the processes are not the same. All processes subserving self-distinction are themselves products of self-production. In contrast, Markov blankets and nonequilibrium steady states in FEP models are there simply by assumption. ${ }^{6}$

Self-distinction is not sufficiently specified by a statement about statistical dependencies or sparsity of connections between variables, nor does it entail any such statement. It is an organizational requirement towards which a set of structural processes contributes. It can result in temporary and selective "barriers" to material, causal, informational, and transformational flows. These are dynamic barriers, actively opening and closing the traffic between interiority and exteriority in ways that are contingently adaptive. They allow or impede the exchange of influences between internal and external processes in a selective manner, rather than operating a blanket statistical mediation between these two realms. Such dynamic barriers are not something that just happens to be the case in living systems. From an enactive point of view, they are necessitated by the precarious operational closure that characterizes autonomous organizations.

\footnotetext{
${ }^{6}$ The assumption is considered self-evident: "Clearly, one needs to differentiate between the system and its environment ... To do this, we have to introduce a third set of states that separates internal from external states. This is known as a Markov blanket." (Ramstead et al. 2018, p. 3-4). This explains the wide applicability of Markov Blankets in examples and models of FEP, sometimes taking the role of boundaries, other times of action and sensory states, and so on. As Raja et al. (2021) argue the choice of where a blanket is located seems to be relatively arbitrary and made to fit the convenience of an FEP interpretation. In an attempt to provide a more principled interpretation of Markov Blankets, Friston (2019) associates them with the structured (ordered) flows in far-from-equilibrium dissipative structures (Nicolis and Prigogine 1977). But he acknowledges that this poses a problem for the FEP formalism, because such structures are in constant material and energetic flux, which contradicts the assumption of a random dynamical attracting set, and confirms that the two central assumptions of FEP are independent, unlike self-production and self-distinction for enaction. Friston considers this problem an unresolved challenge for FEP and its generalization to so-called wandering sets (which would not suffice to address the cases of adaptive parameter and constraint regulation discussed in this section, as these include the possibility of changing sets of variables, such as emerging novel agent-environment relations). The whole formalism, as it currently stands, rests on "the simplifying assumption that over a suitable time scale, blanket states are well defined —as a subset of attracting states" (Friston 2019, 50).
} 
To repeat, self-distinction is not supported structurally only by the formation of organismic boundaries. "Non-boundary" processes of self-distinction include the immune system, CRISPR-Cas anti-virus defense, sweating, shivering and other processes that sustain temperature regulation, processes of maintenance of shape, such as cytoskeletons, musculoskeletal tensegrity, tissue formation and regulation of the extracellular matrix, and so on. Such processes do not entail any particular kind of conditioned independence between internal and external variables; on the contrary, they are regulations of the material encounter between internal and external processes, where internal effects can follow external factors sometimes quite directly.

If we consider "boundary-like" processes of self-distinction, such as cell membranes, the situation is the same. We find a large number of processes that cross "barriers" in both directions. For example, osmosis and other forms of diffusion, the ergodicity-breaking formation of protein nanoclusters affecting ion-channels in neurons (Weron et al. 2017), horizontal transfer of plasmids in bacteria (Soucy et al. 2015), the cumulative effects of diet and adaptation to toxins or their expulsion (Landecker 2011), direct energy transfer in photosynthesis, temperature equilibration in cold-blooded animals, breathing, and "externalized" physiological processes such as the extraction of gaseous oxygen underwater by insects trapping air bubbles in their abdomen (e.g., Turner 2000). Not to mention more complex processes such as cell division, sexual reproduction, host-parasite relations, gut microbiota, or symbiosis in general. The list does not include external processes that are hard or impossible to regulate, such as the effects of ionizing radiation, gravity, or unavoidable physical impacts, but that nevertheless affect internal variables directly. Some of these potential exchanges are avoided or rejected, some are mediated by the activity of the organism, others remain unmediated in the encounter and mediated only in their effects. The condition of operational closure is not lost even in the latter case, provided the effects of an unmediated exchange can be adaptively assimilated (e.g., a fever response to bacterial infection). In none of these cases does it make sense in general to postulate an axiom of conditional statistical independence between internal and external processes. Such a structural barrier is too strong and too undiscriminating to account for the complexity of biological phenomena. Several of these cases we have mentioned violate this axiom. And they are not rare.

Our claim is that membranes and other boundary processes of self-distinction do not generally behave like Markov blankets. Conditional statistical independence introduces a fictitious kind of self- 
distinction, one in which the organism is able to screen and have a say in all of the interactions with the world. By contrast, agency, in the enactive view, involves not just the regulation of boundaries, but also the a posteriori regulation of more direct, boundary-crossing influences of the world, by taking advantage of these influences when useful (e.g.. nourishment) and compensating for their negative effects when not.

To better capture this more subtle idea of how agents relate to their environments, enactivists talk about world-involvement (Di Paolo 2014; Di Paolo et al. 2017). This term is used to stress the fact that agency is always a coordination of bodily and environmental processes in all of their sociomaterial complexity. The world is involved in acts in more than contextual ways, that is, its involvement goes beyond being a source of information. The world is also a material enabler of cognitive acts and may also play constitutive roles in such acts, particularly in contributing to determine their normative aspects (what counts as an act succeeding or failing, what makes an act preferable to another). ${ }^{7}$

The world enables and constitutes embodied agency over and above any information it provides to the cognitive processes of the agent. This is not to say such information is not relevant (provided we describe clearly what we mean by this term, and provided we can establish what counts as the frame of relevance in each case). It means that other, non-informational, aspects of material processes are also enabling and constitutive factors in cognitive acts and in sustaining the autonomy of the agent. This is what world-involvement means. Going on a diet, training for a new skill, and so on-the environmental processes in such activities do not merely inform our bodies about how they should change as a consequence, more often than not they change our bodies directly. Drinking a glass of water quenches our thirst in a way that is different from encountering a useful piece of information. A zero$G$ environment does not inform the body of a cosmonaut that it should grow less dense, it "makes it" do so. By engaging in a novel manual skill, we learn through encountering obstacles to accommodate a new norm that emerges from these material encounters, as in the different ways an

\footnotetext{
7 The distinction between contextual, enabling, and constitutive factors was introduced by De Jaegher et al. (2010) to ask the question of whether the dynamic patterns that we observe in social interactions can (sometimes) be said to be constitutive of social cognitive performance, i.e., whether there is social intelligence in the dynamics of the interaction itself and not just within the interactors. A contextual factor merely modulates a phenomenon under observation; it changes it, but is not required for the phenomenon to exist or to be the kind of phenomenon that it is. An enabling factor is one without which the phenomenon would not occur. A constitutive factor is one without which the phenomenon would not be the kind of phenomenon that it is (see also De Jaegher et al. 2016).
} 
expert potter prepares different kinds of clay. We learn to establish a new frame of relevance as a transformation of agent, environment, and their relation. This relevance frame is necessary to establish what counts as meaningful information (e.g., the cues we used to be indifferent to, but now tell us whether this is good wood for sawing, or a well-prepared clay for shaping a pot, or the age of a wine from its bouquet). Relevance, meaning, norms are irreducibly relational properties. ${ }^{8}$ These material and relational transformations are prior to any informational account we can offer.

The enactive approach offers a theory of sensorimotor mastery based on introducing sensorimotor schemes as integrated flows of coordinated bodily and environmental coupled processes (e.g., the movement of water from a glass into the mouth, the activation of swallowing muscles, its flow within the body, and so on). All traffic across a Markov Blanket, in contrast, is informational (mediated by states of variables that constitute the blanket). This concept leaves out precisely such crucial effects of the world on our bodies, many of which are quite direct. The Markov blanket formalism assumes that such effects have already occurred, such that the norms that determine informational relevance are already in place. Again, these discrepancies between FEP and the enactive approach are not minor. They lead to radically diverging theories of sensorimotor engagements even if they start from acknowledging the importance of embodiment and the relevance of sensorimotor contingencies. For a theory of perception based on free energy minimization, such as Seth's (2014), these embodied factors only play a role in the informational economy of hierarchical predictive models, structuring data, selecting sampling strategies, and generating error-based corrections. Such a proposal remains all in-the-head and dismisses the worldly constituents of perceptual experience by reducing them to error signal generation (Di Paolo 2014). For the enactive approach, in contrast, it is from the material constraints introduced by world-involvement in our action and perception that the norms of such activities arise, including, crucially, what counts or does not count as relevant information for the agent itself and not for the external observer (Di Paolo et al. 2017). Whatever one may think of the merits of these different theories and worldviews, the fact remains that they contrast sharply at a fundamental level (this is sometimes acknowledged by those who would still try to reconcile them; see Clavel Vázquez 2020).

\footnotetext{
${ }^{8}$ On the difficulties of accounting for relational properties within the Markov Blanket formalism see Raja et al. (2021). All of sense-making, by definition, is irreducibly relational (see Thompson, 2007; Di Paolo, 2009).
} 
Although we could continue to examine further implications of the differences between enaction and FEP, we trust that we have now said enough to establish that, at the level of their basic assumptions and the consequences that follow from them, the two approaches are deeply incompatible, contrary to what superficial similarities might suggest.

\section{Conclusions}

It is important that our motivation in this article should not be misconstrued as a general statement against intellectual synthesis or against the cross-fertilization of ideas. Enactivists generally welcome and celebrate such exchanges. Nor is our discussion an exercise in gatekeeping. The enactive approach has undergone important changes over the last decades and will continue to evolve. In part, these changes have resulted from the meeting (sometimes the confrontation) of enactive ideas with other theories, disciplines, and practices. A case in point is the ongoing discussion of the relations between enaction and ecological psychology (Chemero 2009; Di Paolo, Heras-Escribano, Chemero, McGann 2021).

Nevertheless, the process of (inter)theoretical comparison cannot be bypassed or replaced by rhetorical devices, such as stating that some similarities are "straightforward" or "easy to see," or by cherry-picking what to keep and what to ignore about a theoretical framework.

Attempts at discussing the compatibility or complementarity between different theories are initially motivated by what look like interesting similarities and resonances between them. This is fine. But the work of inter-theoretic comparison cannot stay at the level of pointing out these resonances. It requires critical examination to determine whether similarities are only apparent and whether they are not based on misinterpretations. This work demands a deeper and detailed engagement with the assumptions behind the theories being compared, as well as their contexts and goals. Despite its abundance and diversity, the literature defending compatibilities between the FEP and autopoietic and enactive theories has advanced very little beyond bald statements of apparent similarities between these approaches. Such statements remain at step zero of the work that needs to be done. 
In this article, we have shown that once we take the next steps of checking for misinterpretations and comparing fundamental assumptions, the FEP, as it stands formulated today, presents a series of unresolvable tensions with the theories of autopoiesis and enaction. Not only do we find misreadings_-such as confusing statements about organization with statements about structures, or conflating notions of self-distinction with material boundaries and the latter with Markov blanketsbut we also find that at the root of these misreadings lie conflicting assumptions about the constitution of agency and self-individuation in biological and cognitive systems.

Our conclusion is that, for these reasons, the FEP is fundamentally incompatible with the enactive approach. The inter-theoretical tensions and differences we have shown are not minor, but spread respectively through each project as a whole. In particular, we have mentioned that enaction conceives of bodies and sense-making as irreducibly historical (both in the sense of depending on the past and in the sense of changing, or enacting, the present) and co-constituted by the sociomaterial world in ways that go far beyond the processing of information. The centrality of the historical transformation of agents and worlds for the enactive approach is in plain view. It is a direct implication of its classical slogan: Laying down a path in walking (Varela et al. 1991; Thompson 2007). In contrast, the key assumptions of the FEP prevent it from reaching a similar conception of life and mind, as we have shown.

There are other fundamental differences between the two approaches that we have not been able to explore here. For example, there are aspects of the temporality of sense-making and lived experience apart from historicity that can be said to be in tension with notions such as predictions and active inference. Sense-making by its technical definition is future-oriented activity, but this orientation can take a variety of forms, intentional and non-intentional, from vague general states of readiness or alert, to conditions of commitment to action and focus or states of openness at the transition between activities. Of course, sometimes sense-making can also take the form of expectations, inferences, and predictions. But we do not think that the varieties of future orientation reduce to the latter cases only. Establishing this point, however, would require careful conceptual and phenomenological analysis, which we cannot pursue here.

Another difference we have not explored concerns the social dimension. Human bodies and minds, apart from being constitutively historical and diverse, are irreducibly social. Any approach that hopes 
to provide explanations for human cognition must acknowledge this fact and supply the right theoretical articulations to work with it. Enactivists have proposed the concept of participatory sensemaking (De Jaegher and Di Paolo 2007) as the keystone for developing an enactive approach to social, linguistic, and ethical agency. This idea, in our view, is at odds with the notions of blanketed individual persistence of cognitive individuals at the heart of the FEP. In participatory sense-making, actions and intentions become socially constituted and transformative of individual agency; they involve not only other participants but also the relational dynamics of the interaction (De Jaegher et al. 2010). This relational dynamics, we surmise, would not be possible if each interactor was subject to the conditional statistical independence that is forced on them by Markov blankets and the need to remain in a nonequilibrium steady state during the participation process. Again, a full argument, which space prevents us from providing here, would be necessary to establish this point.

We have not dwelled on the question of what motivates researchers to attempt to make the FEP compatible with enaction and autopoiesis. One driving motivation seems to be the need to provide more embodied and less neurocentric interpretations of free energy minimization and active inference. Although we approve of this motivation, we think providing this interpretation is an uphill task, in no small measure because of the neurocentric and computational origins of FEP and predictive processing. A lot of intellectual effort in the literature that we have discussed is spent, almost apologetically, in revisionist attempts at reinterpreting computationally loaded ideas, such as model, inference, and prediction, in terms of embodiment, dynamics, and agent-environment couplings. Having a theoretical approach at hand, such as enaction, that already starts from an embodied and situated perspective could perhaps have given the impression that the uphill task was achievable by establishing a few bridges between the theories. We have argued that this is not possible. Whether there are other ways to make the FEP coherently more embodied is not a question we have set ourselves to answer here.

\section{References}

Adolph, K. E. and Hoch. J. E. (2019). Motor development: Embodied, embedded, enculturated, and enabling. Annual Review of Psychology, 70, 141-164. 
Allen, M. and Friston, K. J. (2018). From cognitivism to autopoiesis: Towards a computational framework for the embodied mind. Synthese, 195, 2459-2482, doi:10.1007/s11229-016-1288-5.

Anderson, M. L. (2014). After Phrenology: Neural Reuse and the Interactive Brain. Cambridge, MA: MIT Press.

Anderson, M. L., Richardson, M. J., and Chemero, A. (2012). Eroding the boundaries of cognition: implications of embodiment. Topics in Cognitive Science, 4, 717-730. doi: 10.1111/j.17568765.2012.01211.x.

Ashby, W. R. (1962). Principles of the self-organizing system. In Principles of Self-Organization: Transactions of the University of Illinois Symposium, H. Von Foerster and G. W. Zopf, Jr. (eds.), Pergamon Press: London, UK, pp. 255-278.

Baltieri M., Buckley C. L. and Bruineberg, J. (2020). Predictions in the eye of the beholder: an active inference implementation of the Watt governor, Artificial Life Conference, 2020. 121-129

Beer, R. D. (2015). Characterizing autopoiesis in the Game of Life. Artificial Life, 21(1),1-19. doi:10.1162/ARTL_a_00143.

Beer, R. D. (2020). Bittorio revisited: Structural coupling in the Game of Life. Adaptive Behavior, 28(4), 197-212. doi: 10.1177/1059712319859907

Biehl, M., Pollock, F. A., and Kanai, R. (2021) A technical critique of some parts of the Free Energy Principle. Entropy, 23, 293. https://doi.org/10.3390/e23030293.

Bitbol, M. and Luisi, P. L. (2004). Autopoiesis with or without cognition: defining life at its edge. J. R. Soc. Interface, 1, 99-107 doi: 10.1098/rsif.2004.0012.

Bourgine, P. and Stewart, J. (2004). Autopoiesis and cognition. Artificial Life, 10(3), 327-345. doi: $10.1162 / 1064546041255557$. 
Bruineberg, J. and Hesp, C. (2018). Beyond blanket terms: Challenges for the explanatory value of variational (neuro-)ethology: Comment on "Answering Schrödinger's question: a free-energy formulation”, Physics of Life Reviews, 24, 37-39. doi: 10.1016/j.plrev.2017.11.015.

Bruineberg, J., Kiverstein, J. and Rietveld, E. (2018). The anticipating brain is not a scientist: the freeenergy principle from an ecological-enactive perspective. Synthese, 195, 2417-2444. doi: 10.1007/s11229-016-1239-1.

Chemero, A. P. 2009. Radical Embodied Cognitive Science. Cambridge, MA: MIT Press.

Clark, A. (2015). Radical predictive processing. The Southern Journal of Philosophy, 53(1), 3-27.

Clark, A. (2017). How to knit your own Markov blanket: Resisting the second law with metamorphic minds. In T. Metzinger and W. Wiese (Eds.). Philosophy and Predictive Processing: 3. Frankfurt am Main: MIND Group. doi: 10.15502/9783958573031.

Clavel Vázquez, M. J. (2020). A match made in heaven: predictive approaches to (an unorthodox) sensorimotor enactivism. Phenomenology and the Cognitive Sciences, 19, 653-684. doi: 10.1007/s11097-01909647-0.

Colombo, M., and Wright, C. (2018). First principles in the life-sciences: The free-energy principle, organicism and mechanism. Synthese. Advance online publication. doi:10.1007/s11229-018-01932-w.

Constant, A., Clark, A., and Friston, K. J. (2021). Representation wars: Enacting an armistice through active inference. Frontiers in Psychology, 11, 598733. doi: 10.3389/fpsyg.2020.598733.

De Jaegher, H. (2019). Loving and knowing: reflections for an engaged epistemology. Phenomenology and the Cognitive Sciences, Online first, doi:10.1007/s11097-019-09634-5.

De Jaegher, H., and Di Paolo, E. A. (2007) Participatory sense-making: An enactive approach to social cognition. Phenomenology and the Cognitive Sciences, 6(4), 485-507. 
De Jaegher, H., Di Paolo, E. and Adolphs, R. (2016). What does the Interactive Brain Hypothesis mean for social neuroscience? A dialogue. Philosophical Transactions of the Royal Society B, 371, 20150379. doi: $10.1098 /$ rstb.2015.0379.

De Jaegher, H., Di Paolo, E. A., and Gallagher, S. (2010). Can social interaction constitute social cognition? Trends in Cognitive Sciences, 14, 441-447.

Di Paolo, E. A. (2005). Autopoiesis, adaptivity, teleology, agency. Phenomenology and the Cognitive Sciences, 4, 429-452.

Di Paolo, E. A. (2009). Extended life. Topoi, 28, 9-21.

Di Paolo, E. A. (2014). The worldly constituents of perceptual presence. Frontiers in Psychology, 5, 450.

Di Paolo, E. A. (2018). The enactive conception of life. In A. Newen, S. Gallagher, L. de Bruin (eds), The Oxford handbook of cognition: Embodied, embedded, enactive and extended, (pp 71-94). Oxford University Press.

Di Paolo, E. A. (2020). Enactive becoming. Phenomenology and the Cognitive Sciences, Online first. https://doi.org/10.1007/s11097-019-09654-1.

Di Paolo, E. A., Buhrmann, T., and Barandiaran, X. E. (2017). Sensorimotor life: An enactive proposal. Oxford: Oxford University Press.

Di Paolo, E. A., Cuffari, E. C., and De Jaegher, H. (2018). Linguistic bodies: The continuity between life and language. Cambridge, MA: MIT Press.

Di Paolo, E. A., Heras-Escribano, M., Chemero, A., McGann, M., (eds). (2021). Enaction and Ecological Psychology: Convergences and Complementarities. Lausanne: Frontiers Media SA. doi: 10.3389/978-2-88966431-3. 
Di Paolo, E. A. and Thompson, E. (2014). The enactive approach. In L. Shapiro (Ed.), The Routledge Handbook of Embodied Cognition (pp. 68-78). London: Routledge.

Fischer, K. W., and Bidell, T. R. (2006). Dynamic development of action, thought, and emotion. In W. Damon and R. M. Lerner (Eds.), Handbook of Child Psychology, Vol. 1: Theoretical Models of Human Development (6th ed., pp. 313-399). New York: Wiley.

Friston, K. (2011). Embodied inference: Or "I think therefore I am, if I am what I think.". In W. Tschacher and C. Bergomi (Eds.), The implications of embodiment (cognition and communication) (pp. 89-125). Exeter: Imprint Academic.

Friston, K. (2012). A free energy principle for biological systems. Entropy, 14, 2100-2121. doi:10.3390/e14112100.

Friston, K. (2013). Life as we know it. Journal of the Royal Society: Interface, 10, 20130475. https://dx.doi.org/10.1098/rsif.2013.0475.

Friston, K. (2019). A free energy principle for a particular physics. arXiv, 1906.10184. https://arxiv.org/abs/1906.10184.

Friston, K., Da Costa, L., and Parr, T. (2020). Some interesting observations on the free energy principle. arXiv: 2002.04501. https://arxiv.org/abs/2002.04501

Friston, K., Kilner, J., and Harrison, L. (2006). A free energy principle for the brain. Journal of physiology, $100,70-87$.

Fuchs, T. (2017). Ecology of the brain: The phenomenology and biology of the embodied mind. Oxford University Press.

Hohwy, J. (2013). The Predictive Mind. London: Oxford University Press. 
Hutto, D., and Myin, E. (2013). Radicalizing enactivism: Basic minds without content. Cambridge, MA: MIT Press.

Kirchhoff, M. D. (2018). Autopoiesis, free energy, and the life-mind continuity thesis. Synthese, 195, 2519-2540, doi:10.1007/s11229-016-1100-6.

Kirchhoff, M. and Froese, T. (2017). Where there is life there is mind: In support of a strong life-mind continuity thesis. Entropy, 19, 169.

Kirchhoff, M., Parr, T., Palacios, E., Friston, K., and Kiverstein, J. (2018). The Markov blankets of life: Autonomy, active inference and the free energy principle. Interface, 15, 20170792. doi:10.1098/rsif.2017.0792.

Kiverstein, J. (2020). Free energy and the self: An ecological-enactive interpretation. Topoi, 39, 559574. doi:10.1007/s11245-018-9561-5.

Kostrubiec, V., Zanone, P.-G., Fuchs, A., and Kelso, J. A. S. (2012). Beyond the blank slate: Routes to learning new coordination patterns depend on the intrinsic dynamics of the learner-experimental evidence and theoretical model. Frontiers in Human Neuroscience 6, 222. doi: 10.3389/ fnhum.2012.00222.

Korbak, T. (2021). Computational enactivism under the free energy principle. Synthese 198, 2743-2763 https://doi.org/10.1007/s11229-019-02243-4.

Landecker, H. (2011). Food as exposure: Nutritional epigenetics and the new metabolism. Biosocieties, 6(2), 167-194.

Litwin, P. and Miłkowski, M. (2020). Unification by fiat: Arrested development of predictive processing. Cognitive Science 44, e12867.doi: 10.1111/cogs.12867. 
Longo, G. and Montévil, M. (2013). Perspectives on Organisms: Biological time, Symmetries and Singularities. New York: Springer.

Maturana, H., and Varela, F. J. (1980). Autopoiesis and Cognition: The Realization of the Living. Dordrecht, The Netherlands: D. Reidel.

Maturana, H., and Varela, F. J. (1987). The Tree of Knowledge: The Biological Roots of Human Understanding. Boston: Shambhala.

Molenaar, P. C. (2004). A manifesto on psychology as idiographic science: Bringing the person back into scientific psychology, this time forever. Measurement: Interdisciplinary Research and Perspectives, 2, 201218.

Molenaar, P. C., and Nesselroade, J. R. (2014). New trends in the inductive use of relational developmental systems theory: Ergodicity, nonstationarity, and heterogeneity. In P. C. Molenaar, R. M. Lerner, and K. M. Newell (Eds.), Handbook of Developmental Systems and Methodology (pp. 442-462). New York: Guilford Press.

Nicolis, G. and I. Prigogine, I. (1977). Self-Organization in Nonequilibrium Systems: From Dissipative Structures to Order through Fluctuations, New York: John Wiley and Sons.

Orth. D., van der Kamp. J., Memmert. D. and Savelsbergh. G. J. P. (2017). Creative motor actions as emerging from movement variability. Frontiers in Psychology, 8, 1903. doi: 10.3389/fpsyg.2017.01903.

Parr, T., Da Costa, L., Friston, K. (2019). Markov blankets, information geometry and stochastic thermodynamics. Philosophical Transactions of the Royal Society A, 378, 20190159. http://dx.doi.org/10.1098/rsta.2019.0159.

Pessoa, L. (2014). Understanding brain networks and brain organization. Physics of Life Reviews, 11(3), $400-435$. 
Pezzulo, G., Rigoli, F. and Friston, K. (2015). Active Inference, homeostatic regulation and adaptive behavioural control, Progress in Neurobiology, 134, 17-35. doi: 10.1016/j.pneurobio.2015.09.001.

Raja, V., Valluri, D., Baggs, E., Chemero, A., and Anderson, M. L. (2021). The Markov blanket trick: On the scope of the free energy principle and active inference. [Preprint] http://philsciarchive.pitt.edu/id/eprint/18843 (accessed 2021-03-30).

Ramstead, M. J. D., Kirchhoff, M. D., Constant, A., and Friston, K. (2021). Multiscale integration: beyond internalism and externalism. Synthese, 198, 41-70. doi:10.1007/s11229-019-02115-x.

Rath, D., Amlinger, L., Rath, A. Lundgren, M. (2015). The CRISPR-Cas immune system: Biology, mechanisms and applications. Biochimie, 117, 119-128. doi: 10.1016/j.biochi.2015.03.025.

Rose, L. T., Rouhani, P., and Fischer, K. W. (2013). The science of the individual. Mind, Brain, and Education, 7(3), 152-158.

Seth, A. K. (2014). A predictive processing theory of sensorimotor contingencies: Explaining the puzzle of perceptual presence and its absence in synesthesia. Cognitive Neuroscience, 5, 97-118.

Speelman, C. P. and McGann, M. (2016). Editorial: Challenges to Mean-Based Analysis in Psychology: The Contrast Between Individual People and General Science. Frontiers in Psychology, 7,1234. doi: 10.3389/fpsyg.2016.01234.

Stewart, J., Gapenne, O., and Di Paolo, E. A. (Eds.). (2010). Enaction: A new paradigm for cognitive science. Cambridge, MA: The MIT Press.

Soucy, S., Huang, J. and Gogarten, J. (2015). Horizontal gene transfer: Building the web of life. Nature Reviews Genetics, 16, 472-482. doi:10.1038/nrg3962.

Thelen, E., Corbetta, D., and Spencer, J. P. (1996). The development of reaching during the first year: The role of movement speed. Journal of Experimental Psychology: Human Perception and Performance, 22, 1059-1076. 
Thompson, E. (2007). Mind in life: Biology, phenomenology and the sciences of mind. Cambridge, MA: Harvard University Press.

Thompson, E. (2018). Review of Daniel D. Hutto and Erik Myin, Evolving Enactivism: Basic Minds Meet Content, Notre Dame Philosophical Reviews, 2018.01.11. https://ndpr.nd.edu/reviews/evolvingenactivism-basic-minds-meet-content/

Thompson, E. and Stapleton, M. (2009). Making sense of sense-making: Reflections on enactive and extended mind theories. Topoi 28, 23-30 https://doi.org/10.1007/s11245-008-9043-2.

Turner, J. S. (2000). The Extended Organism: The Physiology of Animal-Built Structures. Cambridge, MA: Harvard University Press.

van Es, T. (2020). Living models or life modelled? On the use of models in the free energy principle. Adaptive Behavior, [online first]. https://doi.org/10.1177/1059712320918678.

van Es, T. and Kirchhoff, M. (2021). Between pebbles and organisms: Weaving autonomy into the Markov blanket. Synthese, [online first]. doi: 10.1007/s11229-021-03084-w.

Varela, F. J. (1979). Principles of Biological Autonomy. New York: North Holland.

Varela, F. J. (1991). Organism: A meshwork of selfless selves. In: Tauber A.I. (eds) Organism and the Origins of Self. Boston Studies in the Philosophy of Science, vol 129. Springer, Dordrecht. (pp. 79_ 107). https://doi.org/10.1007/978-94-011-3406-4_5

Varela, F. J. (1992). Making it concrete: Before, during and after breakdowns. Revue Internationale de Psychopathologie, 4, 435-450.

Varela, F. J. (1994/2011). Preface to the second edition of "De Máquinas y Seres Vivos - Autopoiesis: La organización de lo vivo”. Systems Research and Behavioral Science, 28, 601-617. doi: 10.1002/sres.1122 
Varela, F. J., Lachaux, J. P., Rodriguez, E., and Martinerie, J. (2001). The brainweb: Phase synchronization and large-scale integration. Nature Reviews Neuroscience, 2(4), 229-239.

Varela, F. J., Thompson, E., and Rosch, E. (1991). The embodied mind: Cognitive science and human experience. Cambridge, MA: MIT Press.

Veissière, S., Constant, A., Ramstead, M., Friston, K., and Kirmayer, L. (2020). Thinking through other minds: A variational approach to cognition and culture. Behavioral and Brain Sciences, 43, E90. doi:10.1017/S0140525X19001213

Villalobos, M. and Ward, D. (2015). Living systems: Autonomy, autopoiesis and enaction. Philosophy \& Technology, 28, 225-239. doi: 10.1007/s13347-014-0154-y.

Walsh, K. S., McGovern, D. P., Clark, A., and O'Connell, R. G. (2020). Evaluating the neurophysiological evidence for predictive processing as a model of perception. Annals of the New York Academy of Sciences. https://doi.org/10.1111/nyas.14321.

Wiese, W. and Friston, K. J. (2021). Examining the continuity between life and mind: Is there a continuity between autopoietic intentionality and representationality. Philosophies, 6, 18. https://doi.org/10.3390/ philosophies6010018.

Weron, A., Burnecki, K., Akin, E. J., Solé, L. Balcerek, M., Tamkun, M. M. and Krapf, D. (2017). Ergodicity breaking on the neuronal surface emerges from random switching between diffusive states. Scientific Reports, 7, 5404. doi:10.1038/s41598-017-05911-y. 